\title{
Technological Aspects of Semiconductor Thermogenerator (TEG) Assembly
}

\author{
Krzysztof Witek, Agata Skwarek, Beata Synkiewicz, Piotr Guzdek, and Aneta Arazna
}

\begin{abstract}
The processing of waste heat harvested from industrial or domestic installations directly converted into electrical energy using thermoelectric generators (TEG) is an interesting and future-proof solution. The main advantages of these types of generators are the absence of moving, defective parts and very long durability, their main disadvantage - the low efficiency. The generator is a serial connection of up to tens of p-n thermocouples, through which the current of as many as dozen amps can flow. Its quality and reliability depends not only on semiconductor parameters, but also on other structural materials such as ceramics, metals or solder alloys. This paper presents technical findings of research into a semiconductor PbTe thermogenerator(TEG) model. It involves an analysis of possible defects in assembly and their influence on thermogenerator. Ways of mitigating such defects are also discussed.
\end{abstract}

Index Terms - Thermogenerator TEG, solder joints, ceramic elements.

\section{INTRODUCTION}

Thermoelectric generators are devices which convert a heat flow stream directly into electrical energy. They use a phenomenon called Seebeck or thermoelectric effect" discovered in 1921. Early thermocouples were metallic, but modern thermoelectric devices are made from p-type and n-type semiconductor elements (pellets) serially connected by metallic connectors. The Seebeck coefficient $\mathrm{S}$ of a material, usually expressed in $\mu \mathrm{V} / \mathrm{K}$, specifies the magnitude of an thermoelectric voltage in response to a temperature difference across that material. The $S$ coefficient of good-quality thermoelectric materials is at the level of a few hundred of $\mu \mathrm{V} / \mathrm{K}$. The main advantages of these types of generators are the absence of fast-wearing and damage-prone moving parts, silent operation, reliability and decades durability. Their main drawback is low single figure percentage efficiency. However, recently there were reports of the development of new thermoelectric materials with a Figure of Merit (ZT) at a level of 2.2 and higher, which may facilitate the development of thermogenerators with efficiency at a level of 15 to $20 \%$. This significantly broadens the application opportunities [1].

Commercially available basic modules usually have a

Manuscript received April 16, 2013; revised June 28, 2013. The study was partially supported by the European Union within the European Regional Development Fund, through the Innovative Economy grant no POIG.01.01.02-00-108/09 (MIME).

Krzysztof Witek, Agata Skwarek, Beata Synkiewicz, and Piotr Guzdek are with Institute of Electron Technology, Zablocie 39, 30-701, Krakow, Poland.

Aneta Arazna is with Tele and Radio Research Institute, Ratuszowa 11, Warsaw, Poland rectangular shape with a square base and a surface, depending on the power, from a few to tens of $\mathrm{cm} 2$. They are made of semiconductor thermoelectric materials (usually the alloys Bi2Te3 and Sb2Te3). The types of component materials from which the thermogenerators are formed along with their size determines the operating temperature range and their performance characteristics (eg, efficiency, power). The electrical parameters are also dependent on the number of pairs of semiconductors, which in one module, depending on its power, can range from a few dozen to a few hundred. Commercially available low power generators are characterized by the following parameters: module surfaces $10 \div 50 \mathrm{~cm} 2$, power range $2 \div 20 \mathrm{~W}$, output voltage range $2 \div 8$ VDC, maximum continuous working temperature $+3000 \mathrm{C}$. The estimated unit price is about $3 \div 10 \$ / \mathrm{W}$, plus additional accessory costs (heatsink, fan, conductive lubricant, etc.), which often exceed the cost of the module.

As the above data shows, single thermomodules have relatively poor electrical parameters. In order to improve them, combinations of a series of parallel module electrical connections are used. In addition, the application of high temperature using heat sources such as burners to natural or liquefied gas can produce rated power of up to several $\mathrm{kW}$ at rated voltages up to 30VDC [2], [3].

Because of their advantages, such as their small size, durability and reliability, thermoelectric generators where the heat source is the natural decay of radioactive elements (Radioisotope Thermoelectric Generator RTG) are widely used as power sources in space technology (eg the Curiosity probe, which currently exploring the surface of Mars). In this case, the following semiconductors are used as the thermoelectric materials: $\mathrm{PbTe}-$ working temperature up to $+900^{\circ} \mathrm{C}$ or $\mathrm{SiGe}$-working temperature up to $+1500^{\circ} \mathrm{C}$ [4].

Currently, many laboratories are carrying out research on the use of thermogenerators to convert waste heat produced in industrial plants or in the exhaust systems of cars. Such devices could be used to supply of small residential buildings heated by fireplaces or installation based on liquid propane gas in cars, caravans or to power small and medium-sized boats. The widespread introduction of thermoelectric generators could lead to a much better use of the energy produced, a reduction of fuel consumption and thus of exhaust gas emissions. Currently, a wide application of thermogenerators in practice is limited by their low efficiency, but intensive research in this area in progress and gives hope for a quick improvement in the present state of affairs. The enormous progress in electronics that has been made in recent years, for example, the improvement of LED efficiency, has led to the belief that in the near future, the 
energy obtained from waste heat sources will become an important alternative source of electrical energy.

\section{A. Basic Problems of Thermogenerator Assembly}

Generally, it can be concluded that the thermogenerators are devices designed for reliable, long-term operation in difficult environmental conditions, particularly at high ambient temperatures and with large temperature gradients between cold and hot plates. In addition, the generator is a serial connection with even tens of $p-n$ thermocouples, through which the current of as many as dozen amps can flow. The main goal of the generator is the minimization of series resistance, in practice even to the level of single mohms, and consequently to a minimization of the Joule heat which is dissipated on $\mathrm{p}-\mathrm{n}$ pellets. These requirements determine the practical application of specific material, design and technological solutions. For example, due to their high thermal resistance, materials such as ceramics, metals or cermet pastes, are preferred to plastics. For connecting the various generator parts, for construction reasons and because of the necessity to achieve, low joint resistance is the best to use soldering or brazing technologies. The selection of suitable alloy compositions with regard to their melting points is essential.

The reliability of electronic systems depends on solder joint quality. In the case of the soldering of ceramic elements, the problem good joint quality is even greater. Ceramics can not be wetted in a classical way. The wettability level, defined as the ability of a surface to be coated with a thin, uniform and continuous layer of liquid solder, strongly influences the stability of a solder joint. The connection of ceramics and metal is based on the fusion of a glaze into the ceramics pores.

Additionally the CTE mismatch of ceramics and metal layers is greater than in case of soldering where there is a glass-epoxy substrate. Incorrect selection of connected elements with different CTE coefficients may lead to an increase in internal stresses, for example under the influence of the temperature gradient. This results in a semiconductor totally cracking or breaking the joint's continuity. The soldering of thermoelements of a relatively large size encourages even more problems. Improper dispensing of solder the paste may lead to the formation of redundant solder outside the solder pads or to the creation of voids inside solder joints.

\section{EXPERIMENTAL}

To estimate the possibility of defects in solder joints used for the jointing of ceramics elements test samples were prepared. Thermoelements composed of doped PbMnTe with gold ohmic contacts were soldered on a ceramic substrate (Al2O3) with PtAg pads. The top and bottom ceramic substrates are respectively hot and cold plates. The temperature gradient applied between them induces voltage at the thermogenerator electrical terminals. The unleaded solder paste SAC305 was applied through a stencil of $100 \mu \mathrm{m}$ thickness using the printing method. Vapor phase soldering (VPS) was used as the soldering method. The biggest advantage of this method is that there is no possibility of overheating. VPS uses the latent heat of liquid vaporization to provide heat for soldering. This latent heat is released as the vapor of the inert liquid condenses on component leads and PCB lands. In VPS, the liquid produces a dense, saturated vapor that displaces air and moisture. The temperature of the saturated vapor zone is the same as the boiling point of the vapor phase liquid [5]. It is also possible to solder the samples with vacuum option. In the experiment the soldering temperature was at the level of $230^{\circ} \mathrm{C}$.

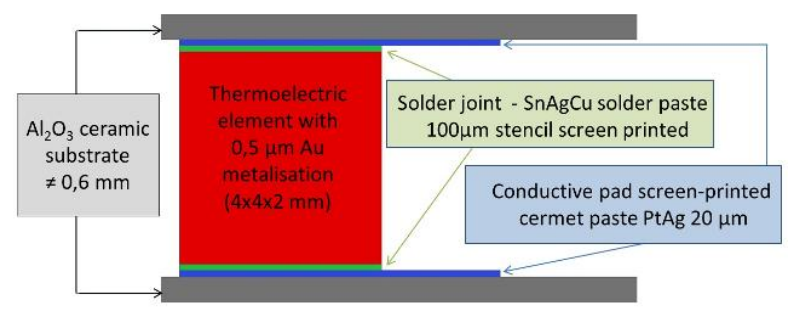

Fig. 1. Thermoelectric element - cross section..

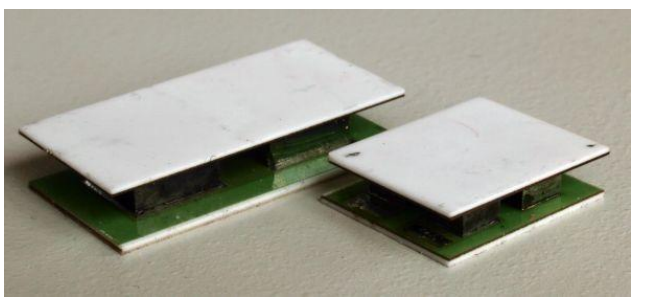

Fig. 2. Models of thermoelectric generators

To check the accuracy of the solder joint, the electrical measurements were done. For the evaluation of solder joint quality optical images and SEM pictures of the cross-section were taken. The cross sections of the samples were made using a metallographic polisher and scanning electron microscope. X-ray images of the solder were taken.

\section{RESULTS}

\section{A. Electrical Measurements}

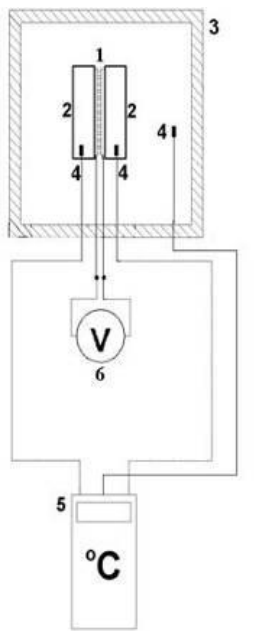

Fig. 3. Measurement system of Seebeck effect (where: 1 - thermoelement, 2 - heaters, 3 - measurement chamber, 4 - temperature sensor, 5 - temperature registrator, 6 - voltage measurement).

The Seebeck effect was measured. For this purpose a measurement system was devised (Fig. 1). Such a system allow allows the measurement of Seebeck effects in a 
temperature range from $-60^{\circ} \mathrm{C}$ to $+180^{\circ} \mathrm{C}$. It is also possible to set a desirable temperature gradient form 0.1 to $5^{\circ} \mathrm{C}$. Exemple results determined for a single $\mathrm{p}$-n thermocouple are presented in Fig. 2. The measured voltage (U) is proportional to the temperature difference $(\Delta \mathrm{T})$ via the Seebeck coefficient $\alpha \mathrm{s}(\mathrm{U}=\alpha \mathrm{s} \Delta \mathrm{T})$.

The temperature dependence of the Seebeck coefficient is shown in Fig. 3. The $\alpha$ s coefficient strongly increases before reaching a maximum at a temperature of $440 \mathrm{~K}$ and then decreasing. A reduction the $\alpha$ s coefficient above $440 \mathrm{~K}$ may be caused by degradation of the gold ohmic contact. The maximum value of $\alpha$ s coefficient $(444 \mu \mathrm{V} / \mathrm{K})$ is higher then the Seebeck coefficient presented for other thermoelectric materials based on lead telluride and bismuth telluride [6], [7].

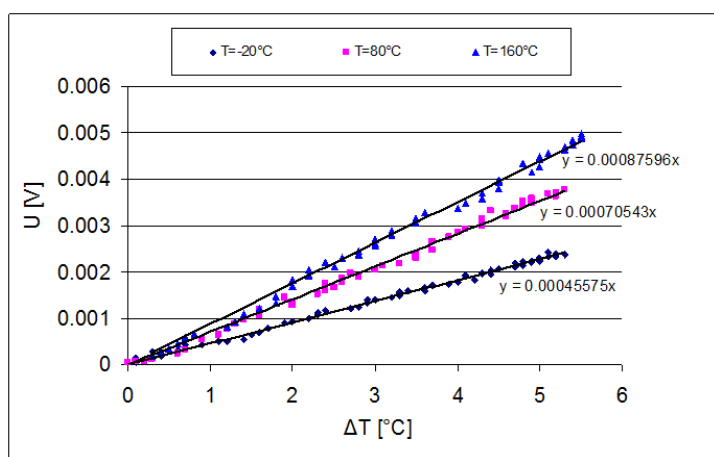

Fig. 4. Dependence of thermoelectric voltage on temperature changes with different thermoelement temperature.

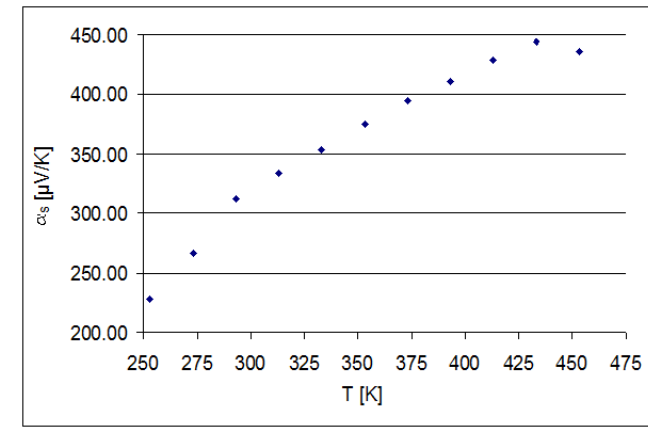

Fig. 5. Dependence of Seebeck effect on temperature for single p-n junction

\section{B. Defects}

\section{1) Paste redundancy}

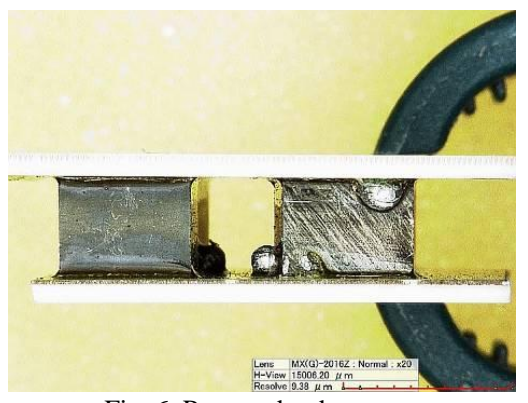

Fig. 6. Paste redundancy.

The dimensions of the thermoelement were $4,0 \times 4.0 \mathrm{~mm}$. Soldering an element of this size encourages problems. The thickness of the solder paste need to be enough to maintain the element, but too thick layer may cause paste redundancy as presented in Fig. 6. Usually, the solder paste is printed on the solder pad by a steel stencil. Its thickness can be different ( for example, 50,100,120, or $150 \mu \mathrm{m}$ ) and therefore the volume of solder can also be different. A excess of paste results in the formation outside of the soldering pads characteristic, spherical spews. This harms the operation of the generator though the partial shorting of pellets. Before starting the assembly of thermogenerators, the thickness of solder paste should be practically determined.

\section{2) Incorrect joints between substrate and thermoelement}

The technological process of generator assembly requires the simultaneous, double-side soldering of many pellets placed between two parallel ceramic plates. Given even slight geometrical differences between the pellets, operating at the same time, and the surface tension force of the molten solder, spontaneous movement and ultimately a permanent shift of the element can occur (Fig. 7). The result may be incorrect joint shape between the element and the solder pad, which can increase joint resistance, as well as causing a lack of parallelism between the hot and cold plate. The use of controlled clamping systems for plate pressure and the simultaneous use of a mechanical grip to prevent components shifting during the soldering process seems to be a preferred, technological solution.

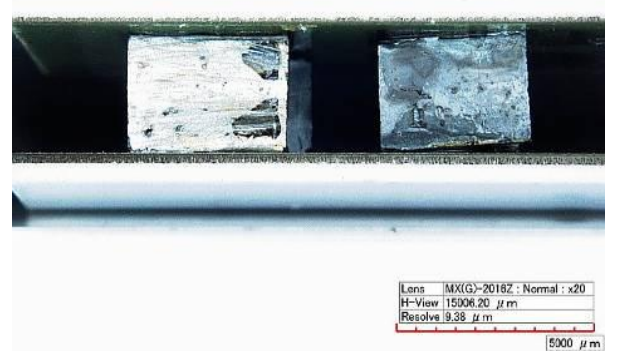

Fig. 7. Spontaneous pellet displacement during soldering

\section{3) Voids in the solder joints}

The phenomenon of voids in solder joints is the formation of empty spaces with rounded shapes in the whole volume of solder. If the voiding is limited in area, it does not cause much damage, although it can increase the resistance of the joints, which may have an adverse impact on their long-term work at overloading. The main reason for the phenomenon is thought to be that the volatile substances (solvents) which evaporate during the soldering process, cannot exhaust completely especially for plane solder joints and closed gaps and will be trapped in the solder joints [8].

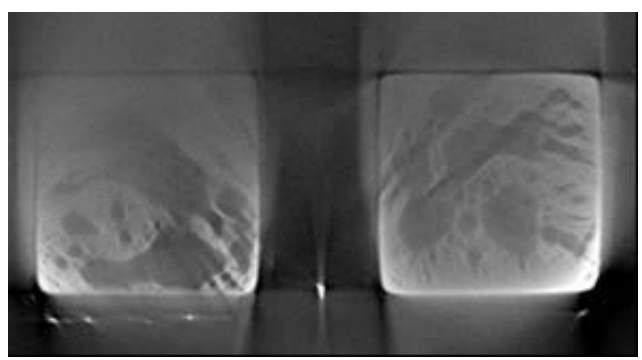

Fig. 8. X-ray analysis of the solder joints

$\mathrm{X}$-ray analysis showed a significant number of voids in the 
solder joint (Fig. 8). In this case the void level reached even $40 \%$. According to the IPC-A-610D an acceptable level of voids in BGA solder joints should be less then $25 \%$ in the area of an X-ray image. However, other literature sources indicate the acceptable level of voids not exceeding 50\% [9].

In practice, many material and technological methods of void reduction are used. These include the use of special, "low void" solder pastes, the application of appropriate technological regimes with special consideration to soldering oven profiles, the design of solder pads shape enabling efficient solvent evacuation and the use of vacuum VPS technology.

\section{4) Impurities of solder joints}

The EDS analysis of the cross-section showed that the solder layer is really nonuniform. A high precipitation of gold has been found in the whole area of the layer. No intermetallic compounds such as $\mathrm{Ag}_{3} \mathrm{Sn}$ to ensure durability of the connection were found. The protrusions of Al probably come form polishing powders and they have great influence on solder quality. Reducing of organic and inorganic impurities in solder joints is a very important factor, especially as regards their long-term operation

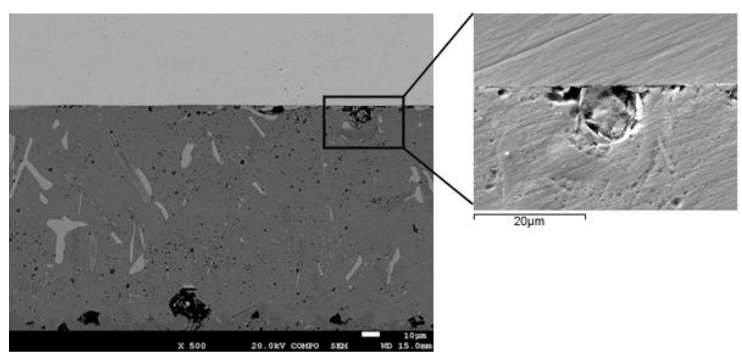

Fig. 9. EDS analysis of the solder joints cross-section

\section{CONCLUSIONS}

Thermogenerator models composed of doped PbMnTe p-n thermocouples with gold ohmic contacts soldered on ceramic substrate $\left(\mathrm{Al}_{2} \mathrm{O}_{3}\right)$ with PtAg pads were characterized by a relatively high $(444 \mu \mathrm{V} / \mathrm{K})$ Seebeck coefficient in the temperature range of $-60^{\circ} \mathrm{C}$ to $+180^{\circ} \mathrm{C}$. Soldering or brazing should be considered as the optimal methods for thermocouple connecting, although is related to the possibility of many different defects.

\section{REFERENCES}

[1] J. Happich. (September 2012). Thermoelectric material expected to convert 15 to $20 \%$ of waste heat to useful electricity. Technology News [Online]. Available: http://www.electronics-eetimes.com

[2] J. Moczygemba. Energy Harvesting TEG Power Strap for Industrial, Chemical, Oil and Gas Application. Marlow Industries [Online] Available:

http://ebookbrowse.com/evergen-power-strap-white-paper-pdf-d3662 17243

[3] Thermoelectric gas generators. RIF Corporation. [Online]. Available: http://eng.rifcorp.ru/products/2/3

[4] A. Jaroszewicz, "Źródła energii elektrycznej na pokładach statków kosmicznych," Systems: Journal of transdisciplinary systems science, vol. 9, no 2/1, pp. 389-401, 2004.

[5] R. Prasad. SMT- Vapor Phase Soldering: The Comeback Kid. Ray Prasad Consultancy Group. [Online]. Available: http://www.rayprasad.com/smt-vapor-phase-soldering-the-comebackkid

[6] Q. Zhang, H. Wang, Q. Zhang, W. Liu, B. Yu, H. Wang, D. Wang, G. $\mathrm{Ni}, \mathrm{D}$. Wang, G. Chen, and Z. Ren, "Effect of Silicon and Sodium on Thermoelectric Properties of Thallium-Doped Lead Telluride-Based Materials," Nano Letters, vol. 12, pp. 2324-2330, 2012.

[7] S. K. Mishra, S. Satpathy, and O. Jepsen "Electronic structure and thermoelectric properties of bismuth telluride and bismuth selenide," Journal of Physics: Condensed. Matter, vol. 9, pp. 461-470, 1997.

[8] R. Diehm, M. Nowottnick, and U. Papa. Reduction of Voids in Solder Joints an Alternative to Vacuum Soldering. IPC APEX EXPO Proceedings. [Online]. Available: http://www.ipcoutlook.org/pdf/reduction_voids_solder_joints_ipc.pdf

[9] T. L. Lewis, C. O. Ndiaye, and J. R. Wilcox. Inclusion Voiding in Gull Wing Solder Joints. IPC APEX EXPO Proceedings. [Online]. Available:

www.ipcoutlook.org/pdf/inclusion_voiding_gull_wing_joints_ipc.pdf

Krzysztof Witek was born in Krakow, Poland in September 1950. In 1973 he graduated from the faculty of Industrial Electrotechnics at the AGH University of Science and Technology.

From 2002, he is working in the Institute of Electron Technology in Krakow Division as manager of Implementation and Production Department For over 20 years he directed small-scale production of polymer heaters, laryngophones, printed circuit boards and electronic assembly. From 2000 to 2013 he participated in several training courses in the filed of electronics. He has authored/co-authored over dozen articles published in international scientific journals. He also participated in the development of several models and prototypes, especially for polymer heaters. Actually he acts in the field of microelectronics in particular on the reliability of solder joints in the test circuits and thermogenerators TEG.

He is a member of International Microelectronics and Packaging Society (IMAPS Poland Chapter). 